\title{
SINGLE AND DOUBLE STAR ASTROMETRY WITH THE MARK III INTERFEROMETER
}

\author{
C.A. HUMMEL \\ Universities Space Research Association \\ NRL/USNO Optical Interferometer Project \\ c/o U.S. Naval Observatory - AD 5 \\ 3450 Massachusetts Av. NW, Washington, DC 20392, USA
}

\begin{abstract}
Present construction of large multi-element optical interferometers is based on experience with several pioneering instruments, a very successful one being the Mark III interferometer on Mt. Wilson, California. With it, over the last few years, some 26 spectroscopic binaries were resolved at separations as small as 3 milliarcseconds (mas) and orbital elements have been derived. In addition, the installation of vacuum delay-lines and multi-color fringe-detection allowed the derivation of dispersion-corrected geometrical delays of stars, which were used to determine relative stellar positions over wide angles with a precision of about 20 mas. We present and discuss recent results with respect to the determination of stellar positions, distances, masses, and luminosities.
\end{abstract}

\section{Introduction}

The Mark III Optical Interferometer was operated by the Remote Sensing Division of the Naval Research Laboratory (with funding from the Office of Naval Research) for six years between 1987 and 1992. During this time, the Mark III contributed direct measurements of stellar diameters and of visual orbits of spectroscopic binaries to astrophysical research. The usefulness of an interferometer for astrometry was demonstrated by measuring star positions with better than FK5 accuracy. See Shao et al. (1988) for a description of the Mark III. 
For diameter and binary observations, a variable baseline was used; lengths ranged from $3 \mathrm{~m}$ to $31 \mathrm{~m}$, but only one configuration was used at a time. For the astrometric observations, we used the $12 \mathrm{~m} \mathrm{~N}-\mathrm{S}$ and E-S baselines realized by three high-precision siderostats mounted on massive concrete piers in climate-controlled protective huts. Fringes could be detected on stars as faint as $m_{\mathrm{V}}=5$. Typically, we obtained 150 to 20075 -sec measures of the delay and the fringe visibility amplitude in a night on a list of eight to 15 stars, half of which were small-angular-diameter stars for calibration. Uniform-disk models were fitted to the calibrated visibility data.

\section{Wide angle astrometry}

The geometrical delay $d_{\mathrm{i}}(t)$ for a star i at time $t$ over a baseline $\mathbf{B}$ is related to its position $\mathbf{S}_{\mathbf{i}}(t)$ through

$$
d_{\mathrm{i}}(t)=\mathbf{B} \cdot \mathbf{S}_{\mathbf{i}}(t)+C(t)
$$

This delay can be derived to high precision (typically one to three microns) from the readings of the laser-controlled delay lines of the Mark III, based on the cancellation of the effects of refraction in an interferometer design featuring evacuated pipes, three-channel detection of the fringe position (at $\lambda \lambda 800 \mathrm{~nm}, 550 \mathrm{~nm}$, and $500 \mathrm{~nm}$ ) for the correction of the atmospheric refractive index fluctuations, and an internal white light source for the measurement of path length variations $C(t)$ induced by temperature drifts. Star positions and baseline coordinates are obtained from a least-squares fit to the delay data.

We show here (Fig. 1) the measured corrections to the FK5 catalog positions for a group of twelve stars, as obtained with the Mark III (Hummel et al. 1994b) and with ESA's astrometry satellite HIPPARCOS (M. Perryman, priv. comm.). The latter data were taken from a preliminary solution based on 18 months worth of observations. We note that a degeneracy of the Mark III solution due to the small declination range of the observed stars prevented the determination of "absolute" declinations. Furthermore, the right ascension zero point is arbitrary. The RMS difference between the Mark III and HIPPARCOS positions is about 20 mas. It is somewhat larger than expected from the formal errors of the Mark III results. Probable causes have been identified as unmonitored baseline drifts during the night, mechanical imperfections of the siderostats, and water vapor fluctuations not solved for in the dispersion correction. However, the agreement between the results of these fundamentally different observing techniques is a "proof-of-concept" for astrometric measurements with optical interferometers. 


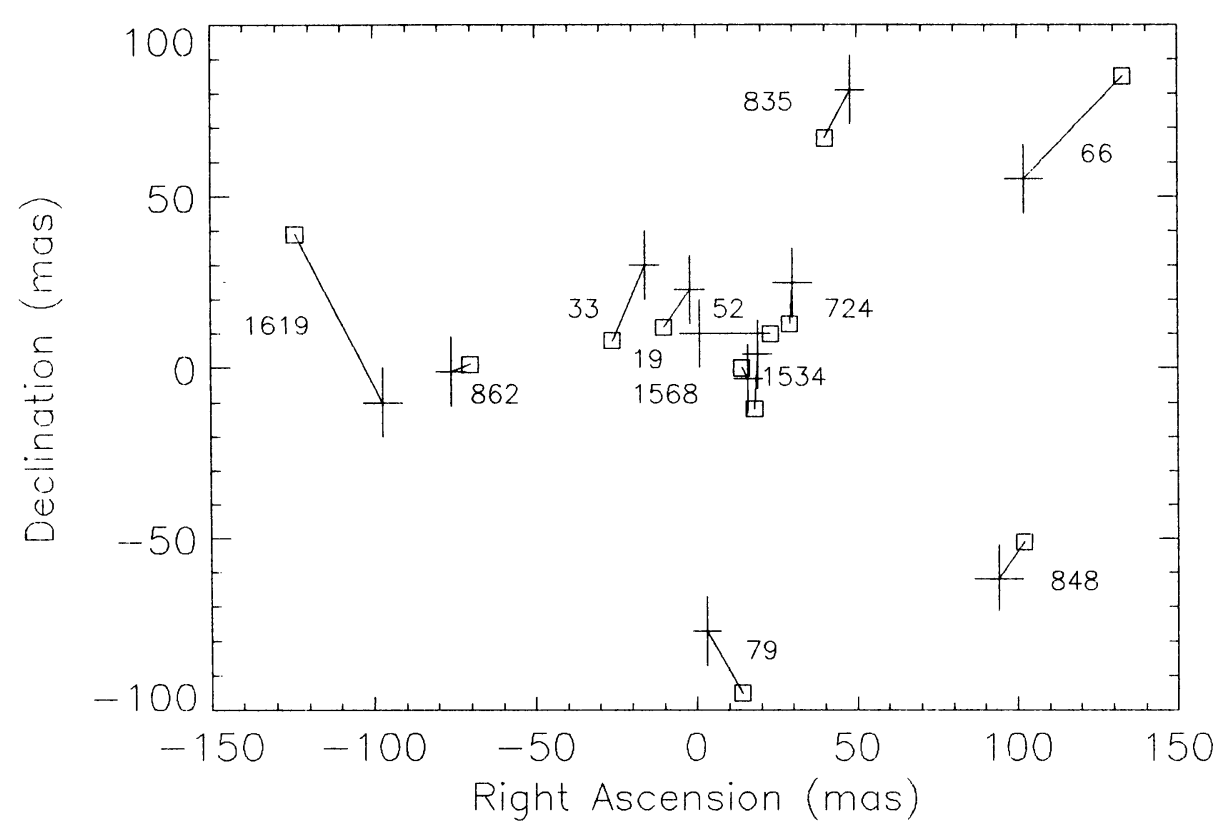

Figure 1. Corrections to the FK5 catalog position for a group of twelve stars. Plus symbols indicate the average results from four years of measurements with the Mark III; square symbols indicate data from the HIPPARCOS satellite.

\section{Binary stars}

Determinations of stellar masses should be better than about $2 \%$ in accuracy and should be accompanied by a determination of the absolute luminosities of the components to help distinguish between stellar evolution models (Andersen 1991). With the Mark III, we were able to resolve orbits of spectroscopic binaries with major axes down to about 3 mas, if the magnitude difference did not exceed about 3.5 . In addition to the orbital elements, we measured the magnitude difference of the components at $\lambda \lambda$ $800 \mathrm{~nm}, 550 \mathrm{~nm}, 500 \mathrm{~nm}$, and $450 \mathrm{~nm}$ (with a $25 \mathrm{~nm}$ bandwidth of each channel). Of the twenty-six spectroscopic binaries which we have resolved and for which we have determined orbital elements, seven are single-lined ( $\pi$ And, 6 Lac, $\tau$ Per, $\alpha$ Dra, $\eta$ Peg, $\alpha$ And, and 113 Her). In six cases, the eccentricity derived from spectroscopy is inconsistent with the Mark III value ( $\chi$ Dra, $\beta$ Tri, $\phi$ Cyg, $\theta^{2}$ Tau, $\xi$ Cep A, and 6 Lac). Thus, we were able to determine masses and absolute luminosities for only a half of the binaries, but in reasonable agreement with recent stellar evolution models. The accuracy for the masses ranges from about $1 \%-4 \%$ (six binaries) to about 10\%-20\% (seven binaries), and is in most cases limited by the accu- 


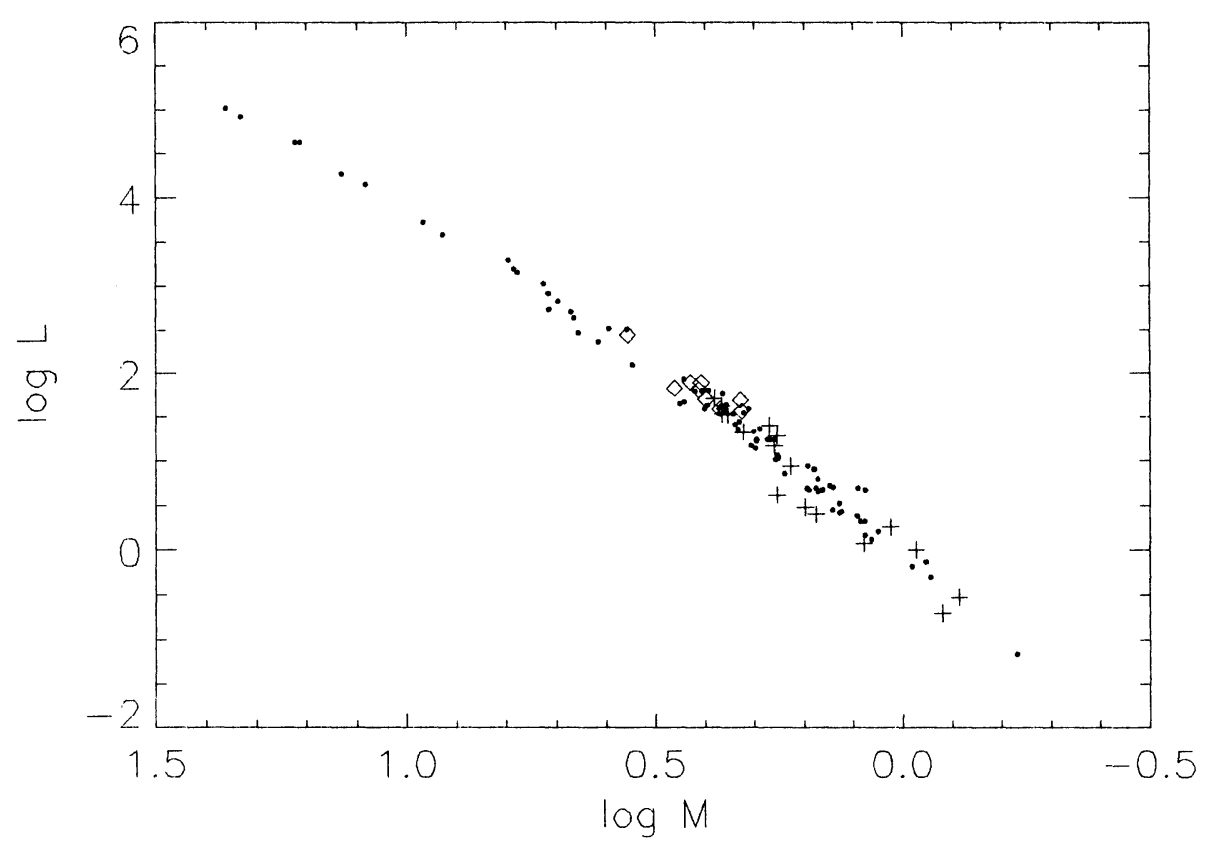

Figure 2. Mass-luminosity diagram for double-lined binaries observed with the Mark III. Plus symbols denote dwarfs or sub-giants; diamonds denote giants. Dots denote eclipsing binary data from Andersen (1991).

racy of the spectroscopy. If the latter were free of errors, mass uncertainties would be accurate to better than $7 \%$ in all cases. In Fig. 2, we plot masses and luminosities for 17 dwarf and 9 giant components, together with data from Andersen (1991) for eclipsing main-sequence binaries. We have not included error bars in the plot due to the large fraction of preliminary orbits. Part of the Mark III results has been published: $\beta$ Ari by Pan et al. (1990), $\alpha$ And by Pan et al. (1992), $\alpha$ Equ by Armstrong et al. (1992a), $\phi$ Cyg by Armstrong et al. (1992b), $\eta$ And by Hummel et al. (1993), $\beta$ Per by Pan et al. (1993), and $\alpha$ Aur by Hummel et al. (1994a).

\section{References}

Andersen, J. (1991), Astron. Astrophys. Rev., 3, 91

Armstrong, J.T., et al. (1992a), Astron. J., 104, 241

Armstrong, J.T., et al. (1992b), Astron. J., 104, 2217

Hummel, C.A., et al. (1993), Astron. J., 106, 2486

Hummel, C.A., et al. (1994a), Astron. J., 107, 1859

Hummel, C.A., et al. (1994b), Astron. J., 108, 326

Pan, X.P., et al. (1990), Astrophys. J., 356, 641

Pan, X.P., et al. (1992), Astrophys. J., 384, 624

Pan, X.P., et al. (1993), Astrophys. J., 413, L129

Shao, M., et al. (1988), Astron. Astrophys., 193, 357 